\title{
A novel age-structured mosquito model for assessing the mechanisms behind vector control success
}

\author{
Emma L Davis ${ }^{1 *}, \mathrm{~T}$ Déirdre Hollingsworth ${ }^{1}$ and Matt J Keeling ${ }^{2}$
}

\footnotetext{
${ }^{*}$ Correspondence:

Emma.Davis@bdi.ox.ac.uk

${ }^{1}$ Big Data Institute, University of Oxford, Waterloo Road, OX3 7LF Oxford, UK

Full list of author information is available at the end of the article
}

\begin{abstract}
Background: Vector control is a vital tool utilised by malaria control and elimination programmes worldwide, and as such it is important that we can accurately quantify the expected public health impact of a range of vector control methods. There are very few previous models that consider vector control induced changes in the age-structure of the vector population and the resulting impact this will have on transmission.
\end{abstract}

Methods: The steady-state solution of a novel age-structured deterministic compartmental model describing the mosquito gonotrophic cycle is analytically derived, with the age of each mosquito measured in the number of gonotrophic cycles (or successful blood meals) completed. From this model we derive analytical expressions for key transmission measures, such as the effective reproductive ratio under control, $R_{c}$, and investigate the impact of commonly used vector control methods on the age-structure of the vector population.

Results: We derive and analyse a novel model with an explicit solution that can be used to directly quantify key transmission statistics and investigate the age-structured impact of vector control. Application of this model confirms current knowledge that adult-acting interventions, such as IRS or LLINs, are highly effective at reducing transmission, particularly in comparison to larvicide usage at the same coverage. We also find that scaling up coverage results in a wider gap in transmission reduction between adult-active and larval-based interventions. For LLINs and IRS the effective reproductive ratio under control, $R_{c}$, decreases exponentially with coverage, whereas for larvicides the relationship is linear. We also find that mid-ranges of LLIN coverage see the largest effect of reduced net integrity on transmission.

Conclusions: Well-maintained, adult-acting vector control measures are substantially more effective than larval-based action at reducing the transmission potential of the mosquito population, particularly at medium to high coverage levels.

Keywords: modelling; mosquito; malaria; vector control; age-structure

\section{Background}

In 2018 there were approximately 228 million cases of malaria worldwide and 405 000 deaths, with children under age 5 accounting for $67 \%$ of all fatalities [1]. Since 20042 billion nets have been distributed to populations at risk of malaria and their usage has been attributed to $68 \%$ of prevented cases in Africa since 2000 [2, 3], particularly in pregnant women and children, in whom bednet usage has more 
than doubled ( $26 \%$ to $61 \%$ ) since 2010 . The majority of these nets are long lasting insecticide treated nets (LLINs) and by 2018 between $40 \%$ and $80 \%$ coverage of the population at risk had been achieved in all 11 high burden to high impact (HBHI) countries.

However, from 2014 to 2018 there has been little change in the incidence rate of malaria, with bednet coverage remaining constant since 2016 [1]. In addition, the use of indoor residual spraying (IRS) has declined, with coverage dropping from $5 \%$ in 2010 to $2 \%$ in 2018 [4]. This may, in part, be linked to reports from 81 countries describing the development of insecticide resistance [5]. In addition, there are requirements to maintain LLIN efficacy through regular distribution, with each net typically lasting for up to 3 years, or 20 washes.

The wide-spread and successful usage of vector control to combat malaria demonstrates the vital role of the mosquito in sustaining transmission. The importance of a deep understanding of how the mechanisms by which these interventions work, as well as the underlying mosquito ecology, has been previously noted $[6,7]$.

Vector control interventions target the mosquito, impacting the feeding cycle and population characteristics. This means that to understand the mechanism through which vector-based interventions reduce transmission, it is first necessary to understand how vector control measures and coverage affect the vector dynamics. The challenges associated with maintaining vector control interventions also mean that it would be useful to understand how transmission changes with the waning efficacy of these interventions over time. Greater characterisation of the changing dynamics of the mosquito population under vector control would also be vital to any future vector-based surveillance (xeno-monitoring) strategy.

A number of widely used models focusing on transmission dynamics have tended to simplify vector dynamics $[8,9]$, whereas models that focus on vector dynamics often do not include transmission [7, 10]. Few previous modelling efforts have combined changes in vector population measures, such as abundance and age-structure, with the resulting impact on transmission [11].

By constructing an age-structured mosquito population model that also includes mosquito infection status, we aim to address this knowledge gap. In particular, we investigate the effect of different vector control measures on the age- and infectionstructure of the vector population and calculate the impact on the reproductive number under control, $R_{c}$, a key transmission measure that can be used to predict long-term elimination or resurgence [12].

Early models of malaria incorporated the mosquito population size, highlighting the importance of the vector to host ratio in determining transmission dynamics [13]. These were extended to derive a formula for the basic reproduction number, $R_{0}$, based on this ratio and a number of other variables, including mosquito feeding and survival rates [14]. Recent models have expanded this further to consider the stages of the feeding cycle $[15,16]$, including vector control interventions at the larval or adult stages but with no age-structure within the adult vector population, with many more models including vector control in some form $[17,18,19,20,21,22$, $23,24]$. However, an independent review of 388 mosquito-borne pathogen models found only 5 models that considered any two-intervention combination of LLINs, IRS and larvicides and only one that considered all three [11]. 
Age-structure has also been modelled explicitly in a number of ways, including splitting the mosquito population into life-cycle stages (egg, larvae, pupae, adult) [25] and focusing on the age-structure of the host population [26, 27]. Partial differential equations (PDEs) have been used to consider adult mosquito age-structure within a transmission model, allowing continuous aging of the mosquito population [28], but this has not been used to compare different vector control measures and their impact on the population structure.

Although vector control methods have long been recognised as important in malaria control and elimination [29], the increasing risk of insecticide resistance and questions around sustainability of interventions have prompted discussions on the best strategy [30,31]. LLINs are the most commonly used measure, but there is also wide-spread usage of IRS and other insecticidal spraying method. Larvicidal methods are often seen as practically more challenging to implement[32], but it has previously been suggested that it may be easier to target spatially-confined larval stages than their highly mobile adult counterparts [30] and that using multiple interventions at different stages of the life-cycle could give improved results [7]. As such, there is a need for detailed analysis of the relative benefits of different vector control methods to inform future decision, in particular modelling can provide insights into whether it is worth exploring larvicidal options further through exhaustive and expensive field-based studies.

\section{Methods}

The analytical solution of a simple age-structured deterministic compartmental model is used to describe the mosquito gonotrophic cycle. The age-structure of the population is measured in the number of gonotrophic cycles, or number of successful blood meals, completed by each individual mosquito. The steady-state of this model is used to investigate the impact of common vector control methods (LLINs, IRS and larvicides) on the age-structure and transmission potential of the vector population.

\section{Gonotrophic cycle model with vector control}

Considering the gonotrophic cycle of an adult mosquito, we divide the stages into four categories: blood-seeking (B), fed (F), gestating (G) and ovipositing (O) [15]. In the absence of intervention new adult mosquitoes are considered to be born into the emerged class at rate $\beta$ and obey a constant natural death rate $g$. Dynamics can then be described using the following system of ordinary differential equations (ODEs):

$$
\begin{aligned}
\frac{d B}{d t} & =\beta(1-\theta)+\pi_{1} O-\pi_{2}\left(q_{1}+q_{2}\right) E-g E \\
\frac{d F}{d t} & =\pi_{2} q_{1} B-\pi_{3} F-g F \\
\frac{d G}{d t} & =\pi_{3}\left(1-q_{3}\right) F-\pi_{4} G-g G \\
\frac{d O}{d t} & =\pi_{4} G-\pi_{1} O-g O
\end{aligned}
$$

where $\pi_{2}$ represents the baseline rate of feeding and moving from blood-seeking to fed; $\pi_{i}, i=1,3,4$, denote the movement between the other states. The magnitude 
of $\beta$ has little bearing on most results as we will generally focus on vector infection prevalence and relative population changes, but it could be chosen to fit a required population size. Similarly $\pi_{i}, i=1, \ldots, 4$ are chosen such that feeding (moving from blood-seeking to fed) is faster than the other transitions; relative values are dependent on the choice of cycle length.

Common notation uses $d, s$ and $r$ to represent the death, success and repelling ratios respectively, but here were use slightly different notation to facilitate ease of mathematical analysis in the results. $q_{1}$ and $q_{2}$ represent the probabilities of vector success or death, respectively, during a feeding attempt and $q_{3}$ is the probability a vector dies after feeding (due to IRS). When no vector control is in use $q_{1}=1$ and $q_{2}=q_{3}=0$. We consider a successful feed to have occurred in any of three potential scenarios: biting indoors despite LLIN or IRS presence; biting indoors in the absence of LLINs or IRS; biting outdoors (taken to occur in proportion $1-Q$, where $Q$ is the probability of a blood meal being taken indoors) - including cattle. Death due to IRS is considered as an additional probability of not surviving between the fed and gestating classes, post feeding and potential transmission. The birth rate is multiplied by a scaling factor $(1-\theta)$, where $\theta=\theta_{0} \hat{\theta}$ is a proportional population reduction due to larvicides; $\theta_{0}$ is the coverage (i.e. proportion of larval sites treated) and $\hat{\theta}$ is the efficacy of the intervention, or proportional reduction in adult mosquitoes emerging from a treated larval site.

The values of $q_{i}, i=1, \ldots, 3$ are given by the following equations, calculated using the feeding dynamics described by Figure 1,

$$
\begin{aligned}
& q_{1}=(1-Q)+Q\left(1-\gamma+\gamma \sigma_{I}\right)\left(1-\omega+\omega \sigma_{L}\right) \\
& q_{2}=Q \omega \nu_{L}\left(1-\gamma\left(1-\sigma_{I}\right)\right) \\
& q_{3}=Q \gamma \nu_{I}
\end{aligned}
$$

with $\omega$ and $\gamma$ representing the coverage of LLINs and IRS respectively. $\sigma_{L}$ and $\nu_{L}$ are the success and death probabilities of feeding in the presence of an LLIN, where $1-\sigma_{L}-\nu_{L}$ is the probability of repeating. $\sigma_{I}$ is the probability of successfully feeding in the presence of IRS, where $1-\sigma_{I}$ is the probability of repeating, and $\nu_{I}$ is the probability of death during the Fed class immediately after exposure to IRS. Values of all parameters are given in Additional File 1: Section 1, Tables S2 and S3.

\section{Age structure}

To gain insight into the age-structure of the vector population we consider a generational formulation of the gonotrophic cycle model, where a subscript $i$ denotes the number of times mosquitoes in a given class have completed the cycle, giving an infinite series of ODEs:

$$
\begin{aligned}
\frac{d B_{i}}{d t} & = \begin{cases}\beta(1-\theta)-\pi_{2}\left(q_{1}+q_{2}\right) B_{i}-g B_{i} & \text { if } i=0 \\
\pi_{1} O_{i-1}-\pi_{2}\left(q_{1}+q_{2}\right) B_{i}-g B_{i} & \text { if } i \geq 1\end{cases} \\
\frac{d F_{i}}{d t} & =\pi_{2} p_{1} B_{i}-\pi_{3} F_{i}-(g+\gamma) F_{i} \\
\frac{d G_{i}}{d t} & =\pi_{3} F_{i}-\pi_{4} G_{i}-g G_{i} \\
\frac{d O_{i}}{d t} & =\pi_{4} G_{i}-\pi_{1} O_{i}-g O_{i} .
\end{aligned}
$$


Births can only occur into generation $i=0$. Using this model it is possible to calculate the parity of the population.

A frequently used assumption in modelling vector borne diseases to assume the vector population is at equilibrium if vector control in a given setting is fixed, as the vector dynamics are faster than the human dynamics. We can hence derive the following relationship between sequential blood-seeking classes:

$$
B_{n}^{*}=K B_{n-1}^{*},
$$

where

$$
K=\frac{\pi_{1} \pi_{2} \pi_{3} \pi_{4} q_{1}}{\left(\pi_{2}\left(q_{1}+q_{2}\right)+g\right)\left(\pi_{3}+g+\gamma\right)\left(\pi_{4}+g\right)\left(\pi_{1}+g\right)}
$$

is a constant and $K<1$ as at equilibrium each generation will be smaller than the previous younger generation, with the newly emerged generation being the largest. This quantity, $K$, can also be interpreted as the gonotrophic cycle survival probability, or the proportion of the vectors that are gravid (have had at least one bloodmeal). As the constant term is less than unity, the difference equation can be solved to get an explicit formula, $B_{n}^{*}=K^{n} B_{0}^{*}$, which can be used to calculate the number of vectors in each feeding generation for initial conditions

$$
B_{0}=\frac{\beta(1-\theta)}{\pi_{2}\left(q_{1}+q_{2}\right)+g}
$$

The proportion of the population that have completed at least one feeding cycle (are parous) is given by

$$
1-\frac{B_{0}}{\sum_{i} B_{i}}
$$

\section{Vector infection model}

We consider a standard SEI model for the vector population with three disease states: susceptible $(S)$, exposed $(Y)$ and infectious $(Z)$. Extending the ODE model (as in Eqns 1-4) to include disease requires sub-dividing each stage of the cycle into these three states, giving a new system of twelve ODEs for each generation, $i$. We assume births only occur in the susceptible population and in generation $i=0$. Assuming a prevalence $x$ in the human population and a probability $c$ that a vector becomes infected after biting an infectious human, then a proportion $x c$ of susceptible vectors moving from blood-seeking to fed become exposed to disease; all exposed mosquitoes can become infected, this occurs at rate $1 / v$ where $v$ is the average vector incubation period. Due to timescales of infection and vector lifespan we do not consider recovery from infection. See Fig. 2 for a diagram of the full model dynamics and Additional File 1: Section 2, Table S3, for the disease parameter values used.

As the host dynamics are slow in comparison to the vector dynamics, we assume that for any change in host prevalence the vector population reaches equilibrium in negligible time. Hence we can use the equilibrium state of the model as an 
approximation for the age and disease distributions for any given human prevalence and use these to calculate transmission measures commonly used in vector-borne disease epidemiology, such as the entomological incoluation rate (EIR), which can be estimated using field data $[33,34]$.

\section{Entomological innoculation rate (EIR)}

The entomological inoculation rate (EIR), $E$, is the expected number of infectious bites received by a single host across a defined time period, described by

$$
E=m a z
$$

where $m$ is the ratio of mosquitoes to humans, $a$ is the blood feeding rate on humans, and $z$ is the fractional prevalence of infectious vectors.

\section{Vectorial capacity}

Vectorial capacity, $V$, denotes the total number of infectious bites that would eventually arise from all the mosquitoes that bite a single infectious human on a single day [35].

$$
V=\frac{m a^{2} p^{v}}{-\ln (p)}=\frac{m a^{2}}{g} e^{-g v}
$$

where $p$ is the vector daily survival probability and $v$ is the extrinsic incubation period in the vector. Alternatively, $g$ is the instantaneous vector death rate.

\section{Basic reproductive number}

From the vectorial capacity we can derive the basic reproductive number, $R_{0}$, for vector borne diseases. This differs from the usual interpretation of $R_{0}$ for nonvector diseases by focusing on the vector dynamics, describing the number of new infectious mosquitoes that would arise from a single infectious mosquito after one parasite generation [35].

$$
R_{0}=\frac{m a^{2} b c}{g r} e^{-g v}=\frac{m a^{2} b c}{-\ln (p) r} p^{v}=\frac{V b c}{r},
$$

where $b$ is the probability a bite from an infectious vector infects a human, $c$ is the probability a bite on an infectious human infects a vector, and $r$ is the human disease recovery rate. Under the assumption that these three parameters are approximately constant, $R_{0}$ is hence linearly proportional to vectorial capacity.

\section{Under vector control}

Vector control interventions impact the vector population size, and hence the mosquito to human ratio, they also affect vector prevalence, feeding cycle length and death rate. Using our model to characterise these relationships for a range of coverages, we can directly calculate the aforementioned transmission measures in the presence of LLINs, IRS or larvicides, as well as any combination of the three (see Additional File 1: Section 3 for analytical derivations and functional forms). 


\section{Results}

The model derivation and analyses outlined above is the major focus of this paper, in particular the explicit quasi-equilibrium solution. We briefly present the impact of interventions and changes in vector dynamics on the age-structure of the vector population and the transmission of disease using these expressions. The model is presented here in the context of malaria, but could easily be extended to consider other mosquito-borne diseases by adjusting the disease-specific parameters.

As has been noted previously, both LLIN and IRS usage have a two-pronged effect on the vector population. Repelling vectors from feeding extends the blood-seeking phase, decreasing the frequency of blood meals per vector. In addition, the insecticidal effects also reduce the total population size by killing vectors either before (LLIN) or after (IRS) feeding. These effects both act to decrease the number of average blood meals per vector per lifetime, hence reducing the likelihood of successful contraction, incubation and transmission of disease. Considering the vector population structure, this manifests as a smaller total population with the age-distribution shifted towards the younger generations (Figure 3).

Conversely, larvicides work by targeting larval stages and hence reducing the adult emergence rate. This results in a reduction in overall population size, but doesn't impact the behaviour or habits of the vectors once they have developed to adulthood, or their individual transmission potential. Our model predicts a linear impact on population size and vector prevalence and doesn't impact the shape of the generational distribution (also Figure 3).

The dual effect of killing and transmission prevention caused by adult-acting interventions, such as IRS or LLINs, scales up with coverage much faster than the population reduction method of larvicides. This translates to an exponential, rather than linear, decrease in the effective reproductive ratio under control with increasing coverage (Figure 4). This pattern is consistent across all transmission intensity settings considered $\left(R_{0}=3.2,32,96\right)$ and it is possible to bring $R_{c}$ below 1 using either IRS or LLINs in all scenarios (see Table ). Perfect (100\%) coverage of larvicides doesn't bring $R_{c}$ below 1 , even for the low intensity setting.

Mid-ranges of LLIN coverage see the largest effect of reduced integrity on the effective reproductive ratio, $R_{c}$, a key measure of transmission (Figure 5). However, the difference between vector population size grows wider as coverage increases. Even at high coverages this type of reduction in efficacy may still undermine program outcomes if $R_{c}$ is close to 1 and new LLINs are not distributed sufficiently frequently.

\section{Discussion}

The model developed here provides an easy-to-use analytical framework for investigating the complex interactions between vector control interventions, age-structured vector population dynamics and disease transmission. It could be extended in a number of ways, including predicting the impact of waning interventions, as demonstrated in Figure 5, or the increase of insecticidal resistance.

Our results demonstrate that LLINs and IRS are more effective than larvicidal interventions, and that the gap in utility increases dramatically with increasing coverage. Analysis of the population age-distribution under the various control measures showed that this is due to the dual effect of population reduction and biting 
prevention, resulting in far fewer infectious mosquitoes in the population. The effect of LLINs and IRS is closely comparable at low to medium coverage, but as coverage increases above 70\% LLINs have an progressively larger impact than IRS on transmission. Additionally, in medium to high transmission settings the linear decrease in $R_{c}$ with increasing larvicidal coverage is insufficient to break transmission if not acting in combination with other measures. In low transmission settings, particularly those where transmission has been brought down artificially through other interventions, larvicides may still be able to play a role in sustaining gains $[36,37]$.

The definition of coverage used (percentage of individuals sleeping under bednets, houses sprayed, or larval breeding sites treated) is difficult to compare across interventions, particularly in terms of the associated costs and feasibilities, as well as adherence measures for LLINs. For larvicidal coverage it would be impossible to find and treat every breeding site if the area considered wasn't very small, and would require regular maintenance throughout a year [38], meaning that achieving high coverage in the terms described here is unrealistic. As coverage of LLINs and IRS is easier to quantify, achieve and maintain, this exacerbates the comparably poor utility of larvicidal methods. However, we have only considered night biting mosquitoes in our analysis and in a setting with high proportions of day biting mosquitoes we would expect LLINs and IRS to have a less effect. There have also recently been some progress in developing slow-release larvicides, with the potential for effects lasting closer to 6 months to a year [39].

We have made a number of additional assumptions about the mosquito biology, including that infection has no impact on vector fitness and that mortality remains constant with age. This second assumption is consistent with current understanding of wild mosquito populations; although senescence is observed in laboratory mosquitoes, wild mosquitoes are expected to die long before they can exhibit any substantial deterioration with age [40]. Our current understanding the first assumption is broadly inconclusive, with conflicting views and results across the literature [41].

Our current model doesn't take account of seasonal changes in vector population sizes and behaviours, which is known to have a substantial effect on malaria transmission [42]. However, as the relative benefit of the three measures is independent of vector population size, our comparisons remain valid. Potentially a low transmission season may mean that intervention efficacy and adherence would be less important during that period, meaning resources could be focused on periods of high transmission.

It is also important to remember that scale-ups in use of insecticides to combat transmission can result in wide-spread insecticide resistance and behavioral changes in sleeping conditions can lead to changes in biting behavior [43, 44]. These factors have the potential to undermine progress made using vector control measures, and in particular evidence of this has been seen in a number of malaria control programs $[45,46,47]$. This is less of a problem for larvicidal interventions, which are less widely used and have a wider range of chemical and biological agents [48]. Settings where resistance has been observed or is feared may benefit from a combination of interventions, in particular larvicides could be used to accelerate gains and delay resistance by slowing the vector birth rate [37]. 


\section{Conclusions}

If well-maintained, adult-acting vector control measures are substantially more effective than larval-based action at reducing the transmission potential of the mosquito population, particularly at medium to high coverage. However, the increasing emergence of insecticide resistance means that it is important to continue investigating new interventions. Larvicides could still be useful as a supplement to other control measures, or in areas where insecticide resistance means that LLINs and IRS have reduced efficacy, but would be insufficient to control transmission if used in isolation in the majority of settings.

Additionally, the model framework developed here is adaptable to different species of mosquito and would be easily extended to consider a number of other mosquitoborne infections through a change of parameterisation. The utility of explicitly describing the impact of different interventions on the vector population size and structure could therefore assist with developing a greater understanding of vector ecology and epidemiology, which could be beneficial in developing future control measures and planning for the impact of insecticide resistance.

\section{Abbreviations}

\section{Declarations}

Ethical approval and consent to participate

Not applicable.

Consent for publication

Not applicable.

Availability of data and materials

All data generated or analysed during this study are included in this published article [and its supplementary information files].

Competing interests

The authors declare that they have no competing interests.

Funding

This work was supported by the Bill \& Melinda Gates Foundation [OPP1184344]. The Bill \& Melinda Gates Foundation had no input into the study design or implementation.

Authors' contributions

MJK and TDH devised the project and main conceptual ideas. MJK over saw the development of the model framework. ELD implemented the model and wrote the manuscript. All authors provided critical feedback and helped shape the research and analysis. All authors read and approved the final manuscript.

Acknowledgements

Not applicable.

Author details

${ }^{1}$ Big Data Institute, University of Oxford, Waterloo Road, OX3 7LF Oxford, UK. ${ }^{2}$ Zeeman Institute, University of Warwick, CV4 7AL Coventry, UK.

References

1. Organization, W.H., et al.: World malaria report 2019 (2019)

2. RBM Partnership to End Malaria: 2 billion mosquito nets delivered worldwide since 2004. https://endmalaria.org/news/2-billion-mosquito-nets-delivered-worldwide-2004

3. Drakeley, C., Lines, J.: In for the long haul: 20 years of malaria surveillance. The Lancet infectious diseases 14(6), 445-6 (2014)

4. Chen, I., Cooney, R., Feachem, R.G., Lal, A., Mpanju-Shumbusho, W.: The lancet commission on malaria eradication. The Lancet 391(10130), 1556-1558 (2018)

5. Rosenthal, P.J., John, C.C., Rabinovich, N.R.: Malaria: How are we doing and how can we do better? The American journal of tropical medicine and hygiene 100(2), 239 (2019) 
6. Ferguson, H.M., Dornhaus, A., Beeche, A., Borgemeister, C., Gottlieb, M., Mulla, M.S., Gimnig, J.E., Fish, D. Killeen, G.F.: Ecology: a prerequisite for malaria elimination and eradication. PLoS medicine 7(8) (2010)

7. White, M.T., Griffin, J.T., Churcher, T.S., Ferguson, N.M., Basáñez, M.-G., Ghani, A.C.: Modelling the impact of vector control interventions on anopheles gambiae population dynamics. Parasites \& vectors 4(1), 153 (2011)

8. Sequeira, J., Louçã, J., Mendes, A.M., Lind, P.G.: Transition from endemic behavior to eradication of malaria due to combined drug therapies: An agent-model approach. Journal of Theoretical Biology 484, 110030 (2020)

9. Bai, Z., Peng, R., Zhao, X.-Q.: A reaction-diffusion malaria model with seasonality and incubation period. Journal of mathematical biology 77(1), 201-228 (2018)

10. Eckhoff, P.A.: A malaria transmission-directed model of mosquito life cycle and ecology. Malaria journal 10(1), 303 (2011)

11. Reiner Jr, R.C., Perkins, T.A., Barker, C.M., Niu, T., Chaves, L.F., Ellis, A.M., George, D.B., Le Menach, A., Pulliam, J.R., Bisanzio, D., et al.: A systematic review of mathematical models of mosquito-borne pathogen transmission: 1970-2010. Journal of The Royal Society Interface 10(81), 20120921 (2013)

12. Smith, D.L., Battle, K.E., Hay, S.I., Barker, C.M., Scott, T.W., McKenzie, F.E.: Ross, macdonald, and a theory for the dynamics and control of mosquito-transmitted pathogens. PLoS pathogens 8(4) (2012)

13. Ross, R.: Report on the Prevention of Malaria in Mauritius. Waterlow, ??? (1908)

14. Macdonald, G., et al.: The epidemiology and control of malaria. The Epidemiology and Control of Malaria. (1957)

15. Killeen, G.F., Govella, N.J., Lwetoijera, D.W., Okumu, F.O.: Most outdoor malaria transmission by behaviourally-resistant anopheles arabiensis is mediated by mosquitoes that have previously been inside houses. Malaria journal 15(1), 225 (2016)

16. Le Menach, A., Takala, S., McKenzie, F.E., Perisse, A., Harris, A., Flahault, A., Smith, D.L.: An elaborated feeding cycle model for reductions in vectorial capacity of night-biting mosquitoes by insecticide-treated nets. Malaria journal 6(1), 10 (2007)

17. Gu, W., Novak, R.J.: Predicting the impact of insecticide-treated bed nets on malaria transmission: the devil is in the detail. Malaria Journal 8(1), 256 (2009)

18. Gu, W., Novak, R.J.: Habitat-based modeling of impacts of mosquito larval interventions on entomological inoculation rates, incidence, and prevalence of malaria. The American journal of tropical medicine and hygiene 73(3), 546-552 (2005)

19. Smith, D.L., Hay, S.I., Noor, A.M., Snow, R.W.: Predicting changing malaria risk after expanded insecticide-treated net coverage in africa. Trends in parasitology 25(11), 511-516 (2009)

20. Chitnis, N., Schapira, A., Smith, T., Steketee, R.: Comparing the effectiveness of malaria vector-control interventions through a mathematical model. The American journal of tropical medicine and hygiene 83(2), 230-240 (2010)

21. Yakob, L., Yan, G.: Modeling the effects of integrating larval habitat source reduction and insecticide treated nets for malaria control. PloS one 4(9) (2009)

22. Killeen, G.F., Knols, B.G., Gu, W.: Taking malaria transmission out of the bottle: implications of mosquito dispersal for vector-control interventions. The Lancet infectious diseases 3(5), 297-303 (2003)

23. Smith, D.L., Dushoff, J., McKenzie, F.E.: The risk of a mosquito-borne infection in a heterogeneous environment. PLoS biology 2(11), 368-368 (2004)

24. Worrall, E., Connor, S., Thomson, M.: A model to simulate the impact of timing, coverage and transmission intensity on the effectiveness of indoor residual spraying (irs) for malaria control. Tropical Medicine \& International Health 12(1), 75-88 (2007)

25. Hancock, P.A., Godfray, H.C.J.: Application of the lumped age-class technique to studying the dynamics of malaria-mosquito-human interactions. Malaria journal 6(1), 98 (2007)

26. Hethcote, H.W., Thieme, H.R.: Stability of the endemic equilibrium in epidemic models with subpopulations. Mathematical Biosciences 75(2), 205-227 (1985)

27. Geisse, K.V., Butler, E.J.M., Cordovez, J.M.: Effects of natural acquired immunity in an age-structured malaria model. Technical report, Technical report, July (2012)

28. Rock, K., Wood, D., Keeling, M.: Age-and bite-structured models for vector-borne diseases. Epidemics 12, 20-29 (2015)

29. Killeen, G.F., Smith, T.A., Ferguson, H.M., Mshinda, H., Abdulla, S., Lengeler, C., Kachur, S.P.: Preventing childhood malaria in africa by protecting adults from mosquitoes with insecticide-treated nets. PLoS medicine 4(7) (2007)

30. Killeen, G.F., Fillinger, U., Knols, B.G.: Advantages of larval control for african malaria vectors: low mobility and behavioural responsiveness of immature mosquito stages allow high effective coverage. Malaria Journa 1(1), 8 (2002)

31. Griffin, J.T., Hollingsworth, T.D., Okell, L.C., Churcher, T.S., White, M., Hinsley, W., Bousema, T., Drakeley, C.J., Ferguson, N.M., Basáñez, M.-G., et al.: Reducing plasmodium falciparum malaria transmission in africa: a model-based evaluation of intervention strategies. PLoS medicine 7(8) (2010)

32. Utzinger, J., Tozan, Y., Singer, B.H.: Efficacy and cost-effectiveness of environmental management for malaria control. Tropical Medicine \& International Health 6(9), 677-687 (2001)

33. Kilama, M., Smith, D., Hutchinson, R., Kigozi, R., Yeka, A., Lavoy, G., Kamya, M., Staedke, S., Donnelly, M., Drakeley, C., et al.: Estimating the annual entomological inoculation rate for plasmodium falciparum transmitted by anopheles gambiae sl using three sampling methods in three sites in uganda. Malaria Journal 13(1), $111(2014)$

34. Smith, D.L., Perkins, T.A., Reiner Jr, R.C., Barker, C.M., Niu, T., Chaves, L.F., Ellis, A.M., George, D.B. Le Menach, A., Pulliam, J.R., et al.: Recasting the theory of mosquito-borne pathogen transmission dynamics and control. Transactions of the Royal Society of Tropical Medicine and Hygiene 108(4), 185-197 (2014)

35. Smith, D., Drakeley, C., Chiyaka, C., Hay, S.: A quantitative analysis of transmission efficiency versus intensity for malaria. Nature Communications 1, 108 (2010) 
36. Derua, Y., Kahindi, S., Mosha, F., Kweka, E., Atieli, H., Zhou, G., Lee, M.-C., Githeko, A., Yan, G.: Susceptibility of anopheles gambiae complex mosquitoes to microbial larvicides in diverse ecological settings in western kenya. Medical and veterinary entomology 33(2), 220-227 (2019)

37. Derua, Y.A., Kweka, E.J., Kisinza, W.N., Githeko, A.K., Mosha, F.W.: Bacterial larvicides used for malaria vector control in sub-saharan africa: review of their effectiveness and operational feasibility. Parasites \& vectors 12(1), 426 (2019)

38. Kroeger, A., Horstick, O., Riedl, C., Kaiser, A., Becker, N.: The potential for malaria control with the biological larvicide bacillus thuringiensis israelensis (bti) in peru and ecuador. Acta Tropica 60(1), 47-57 (1995)

39. Afrane, Y.A., Mweresa, N.G., Wanjala, C.L., Gilbreath III, T.M., Zhou, G., Lee, M.-C., Githeko, A.K., Yan, G.: Evaluation of long-lasting microbial larvicide for malaria vector control in kenya. Malaria journal 15(1), 577 (2016)

40. Ryan, S., Ben-Horin, T., Johnson, L.: Malaria control and senescence: the importance of accounting for the pace and shape of aging in wild mosquitoes. Ecosphere 6(9), 1-13 (2015)

41. Ferguson, H.M., Read, A.F.: Why is the effect of malaria parasites on mosquito survival still unresolved? Trends in parasitology 18(6), 256-261 (2002)

42. Epopa, P.S., Collins, C.M., North, A., Millogo, A.A., Benedict, M.Q., Tripet, F., Diabate, A.: Seasonal malaria vector and transmission dynamics in western burkina faso. Malaria journal 18(1), 113 (2019)

43. Fornadel, C., Norris, L., Glass, G., Norris, D.: Analysis of anopheles arabiensis blood feeding behavior in southern zambia during the two years after introduction of insecticide-treated bed nets. The American Journal of Tropical Medicine and Hygiene 83(4), 848-853 (2010)

44. Weill, M., Lutfalla, G., Mogensen, K., Chandre, F., Berthomieu, A., Berticat, C., Pasteur, N., Philips, A., Fort, P., Raymond, M.: Comparative genomics: Insecticide resistance in mosquito vectors. Nature 423(6936), 136 (2003)

45. Ranson, H., Lissenden, N.: Insecticide resistance in african anopheles mosquitoes: a worsening situation that needs urgent action to maintain malaria control. Trends in Parasitology 32(3), 187-196 (2016)

46. Hemingway, J., Ranson, H., Magill, A., Kolaczinski, J., Fornadel, C., Gimnig, J., Coetzee, M., Simard, F., Roch, D., Hinzoumbe, C., et al.: Averting a malaria disaster: Will insecticide resistance derail malaria control? The Lancet 387(10029), 1785-1788 (2016)

47. Toé, K., Jones, C., N'Fale, S., Ismail, H., Dabiré, R., Ranson, H.: Increased pyrethroid resistance in malaria vectors and decreased bed net effectiveness, burkina faso. Emerging Infectious Diseases 20(10), 1691 (2014)

48. Dusfour, I., Vontas, J., David, J.-P., Weetman, D., Fonseca, D.M., Corbel, V., Raghavendra, K., Coulibaly, M.B., Martins, A.J., Kasai, S., et al.: Management of insecticide resistance in the major aedes vectors of arboviruses: Advances and challenges. PLoS neglected tropical diseases 13(10) (2019)

Figures

Figure 1 Gonotrophic cycle with vector control. A schematic depicting the mosquito gonotrophic cycle model. In the model mosquitoes move from Blood-seeking (B), to Fed ( $F$ ), to Gestating $(\mathrm{G})$, to Ovipositing $(\mathrm{O})$ and back to Blood-seeking. Larvicide usage impacts the emergence, or birth, rate of adult mosquitoes and LLIN and IRS interactions take place between Blood-seeking and Fed.

Figure 2 SEI disease model in mosquitoes. A schematic depicting the SEI (susceptible, exposed, infectious) formulation of the disease model used. Upon successful feeding, mosquitoes become infected with probability $p$ and enter the exposed class. Mosquitoes transition from exposed to infectious at rate $1 / \nu$, where $\nu$ is the extrinsic incubation period of malaria.

Figure 3 Population age-distribution with disease. Bar plots showing the age-distribution of a vector population at equilibrium (total count, indexed by number of gonotrophic cycles completed) with a variety of combinations of vector control interventions. All interventions are assumed to have $50 \%$ coverage. Bars are coloured by the proportion of vectors in each cycle generation that are susceptible (green), exposed (yellow) and infectious (red) for malaria at $40 \%$ host prevalence.

Figure 4 Reproductive ratio, $\boldsymbol{R}_{c}$, by vector control method and coverage. Graphs showing the relationship between $R_{0}$ and coverage for the three main vector control interventions: LLINs (solid), IRS (dashed) and larvicides (dot-dashed). Low (green), medium (blue) and high (red) transmission scenarios are presented. Left: linear y-axis; Right: logarithmic y-axis. 
Figure 5 LLIN integrity comparisons. Graphs showing the relationship between $R_{c}$ (left) and population size (right) and LLIN coverage. Lines represent good condition (6 holes, solid) and poor condition ( 80 holes, dashed) for a mid-to-high estimate of $R_{0}=65$.

Table 1 Vector control coverage required to bring $R_{c}<1$, by transmission intensity

\begin{tabular}{cccc}
\hline & High $\left(R_{0}=96\right)$ & Medium $\left(R_{0}=32\right)$ & Low $\left(R_{0}=3.2\right)$ \\
\hline LLINs & $88 \%$ & $63 \%$ & $21 \%$ \\
IRS & $90 \%$ & $67 \%$ & $20 \%$ \\
\hline
\end{tabular}

Tables

Additional Files

Additional file 1

Additional file 1 contains further details on the parameterisation of the model and derivation of functional forms for the key transmission measures considered. Section 1 describes the data used to parameterise the gonotrophic cycle model and vector control interventions. Section 2 describes the malaria-specific disease parameters used. Section 3 contains details on the derivation of the transmission measures (EIR, $R_{c}$, and vectorial capacity) under vector control interventions. 